\title{
Transmission Delay in Large Scale Ad Hoc Cognitive Radio Networks
}

\author{
Zhuotao Liu \\ Dept. of Elec. Engin. \\ Shanghai JiaoTong Univ. \\ Shanghai, China \\ zhuotaoliu@sjtu.edu.cn \\ Wentao Luan \\ Dept. of Elec. Engin. \\ Shanghai JiaoTong Univ. \\ Shanghai, China \\ Iwt1104@sjtu.edu.cn
}

\author{
Xinbing Wang \\ Dept. of Elec. Engin. \\ Shanghai JiaoTong Univ. \\ Shanghai, China \\ xwang8@sjtu.edu.cn \\ Songwu Lu \\ Dept. of Comp. Sci. \\ Univ. of California, LA \\ Los Angeles, USA \\ slu@cs.ucla.edu
}

\begin{abstract}
There has been recent interest within the networking research area to understand the transmission delay in Cognitive Radio (CR) Networks with overlapping primary network and secondary network. In this paper, we investigate the scaling behavior of transmission delay in large scale ad hoc CR networks. We take different scenarios of CR networks into consideration and thus obtain a wind range of results. We first neglect propagation delay and study the ratio of transmission delay to distance, denoted by $\gamma\left(\lambda_{S}, \mathcal{A}_{P}\right)$, using the Poisson Boolean Model and Poisson Random Connection Model of continuum percolation theory. We show that $\gamma\left(\lambda_{S}, \mathcal{A}_{P}\right)$ is a constant and figure out its exact value in supercritical secondary network. In case of subcritical secondary network, we introduce a multi-cluster hop transmission process to get the lower bound of $\gamma\left(\lambda_{S}, \mathcal{A}_{P}\right)$. Then we take propagation delay into consideration to obtain further results. Finally, we use simulation results to verify our theoretical analysis. The results present the scaling behavior of transmission delay in CR networks and provide the design guidelines for large scale wireless networks.
\end{abstract}

\section{Categories and Subject Descriptors}

C.2.1 [Computer-Communication Networks]: Network Architecture and Design-Wireless Communications

\section{General Terms}

Theory and Performance

\section{Keywords \\ Connectivity, Delay, Cognitive Radio}

Permission to make digital or hard copies of all or part of this work for personal or classroom use is granted without fee provided that copies are not made or distributed for profit or commercial advantage and that copies bear this notice and the full citation on the first page. To copy otherwise, to republish, to post on servers or to redistribute to lists, requires prior specific permission and/or a fee.

MobiHoc'12, June 11-14, 2012, Hilton Head Island, SC, USA.

Copyright 2012 ACM 978-1-4503-1281-3/12/06 ...\$5.00.

\section{INTRODUCTION}

Most of the frequency spectra suitable for wireless communication have already been licensed by the Federal Communications Commission (FCC) in the USA. Today, as wireless communication witnesses a dramatic growth, efficient usage of spectrum becomes necessary. However, recent measurements [4] have shown that over $90 \%$ of the time, a large percentage of the licensed bands remain unused. This under utilization of statically allocated spectra motivates the study of a new type of wireless communication network, the Cognitive Radio (CR) networks, composed of the overlapping primary network and secondary network. By carefully sensing and learning the primary users' presence through their cognitive devices, secondary users can identify and utilize spectrum opportunities while avoiding unacceptable interference to primary users. Therefore, cognitive radio has dramatically improved the spectrum efficiency.

Since the Gupta and Kumar's landmark work [5], the study of wireless networks has received great attention. Besides capacity, coverage [7] and connectivity [8] of wireless networks are also well studied. These works mainly concern the homogenous networks, where nodes are distributed according to Poisson Point Process. However, the heterogeneity of CR networks, caused by the heterogeneity of spectrum opportunities over time and space, makes the study of CR networks different from that of homogenous networks. Specifically, secondary users within the transmission range of any primary users that are broadcasting or receiving information can not have spectrum opportunities. Therefore, the secondary users will not be able to send or receive massages even if they are topologically connected with the outside secondary network. From the perspective of secondary network, it has "vacuum" space. Hence, the initial homogenous Poisson Point Process becomes heterogenous due to the impact of primary network. Therefore, the previous results can not be efficiently applied into CR networks

As CR networks receive more and more attention, some recent works have put them on priority. In [9], L. Ding et al. proposed a routing and spectrum allocation algorithm for CR networks to maximize the throughput. By using percolation theory, especially continuum percolation [1], W. Peng et al. [10] and W. Ren et al. [11] have studied the connectivity in CR networks in terms of density pairs of primary users and secondary users $\left(\lambda_{S}, \lambda_{P T}\right)$. They both show that the overall connectivity exits if and only if $\lambda_{S}>\lambda_{S}^{*}$ and $\lambda_{P T}<\lambda_{P T}^{*}$, where $\lambda_{S}^{*}$ and $\lambda_{P T}^{*}$ are two critical density values. 
When we consider network design and architecture, one of the most significant issues is transmission delay. In [12], S. Bodas et al. have considered the problem of designing scheduling algorithm$\mathrm{s}$ for small delay in multi-channel wireless networks. In [13], Z. Kong et al. have answered what fraction of the network eventually receives the message when the network is subcritical and how long the delay is. In [14], S. Zhao et al. studied the relationship between node density and transmission delay. Again we noticed that these works mainly considered the homogenous networks. This motivates us to answer the following question in this paper:

- How does transmission delay scale with the distance from source to destination in large scale ad hoc Cognitive Radio networks?

To make our analysis meaningful, we first introduce the connectivity of networks, the prerequisite for communication. Full connectivity [6] can ensure the successful communication between any randomly chosen node pairs. However, the huge power consumption of maintaining such strong connectivity makes it impractical in some situations where the total throughput of energy for each node is limited. Thus, it is necessary to introduce a slightly weaker connectivity paradigm, i.e., an infinite connected component containing a high proportion of the networks' nodes exists.

Using the continuum percolation theory, it is possible to achieve this weaker connectivity in large scale networks. The two most general models in [1] are Poisson Boolean Model (BM) and Poisson Random Connection Model (RCM). The two models describe the behaviors of connected component in a random geometric graph in which nodes are distributed according to Poisson Point Process with node density $\lambda$, and two nodes share a link according to a connection function. A fundamental result of RCM or BM points out a phase transition effect in percolation of networks. For $\lambda>\lambda_{c}$ (supercritical), there exists a unique connected component containing an infinite number of nodes, i.e., the networks percolate. For $\lambda<\lambda_{c}$ (subcritical), the unique connected component is broken into infinite number of mutually disconnected finite components almost surely. Thus, the network cannot achieve overall connectivity and messages may not be able to reach the destination if it resides in the different component with the source. Therefore, we need to introduce mobility of nodes into the network as mobile nodes which have been informed the information may meet the source even if they are within different components initially. Consequently, the messages can see a probability to disseminate among the network when nodes are mobile.

We will study the transmission delay under the weaker version of connectivity. Waiting delay and propagation delay ${ }^{1}$ are two major contributions to transmission delay. In supercritical networks, secondary users within the unique infinite component have instantaneous connectivity. Then waiting delay consists only of the waiting time for spectrum opportunities. Whereas in subcritical networks, the waiting delay is composed by the waiting time for both instantaneous connectivity and spectrum opportunities. Usually, such a waiting delay may be in the order of seconds, minutes or even larger time units. As for the propagation delay, it is in the order of milliseconds when the network is not heavily loaded. Therefore, compared to the waiting delay, it is negligibly small. Given spectrum opportunities, messages can disseminate among one connected component instantaneously if ignoring the propagation delay.

In our approach to the scaling behavior of transmission delay, we consider different scenarios of CR networks. First, we neglect the propagation delay and investigate the impact of secondary user's

\footnotetext{
${ }^{1}$ As is usual with percolation-theory based analysis for delay, the queueing delay in the transmission of messages is not accounted.
}

density and primary network on the transmission delay in supercritical secondary network. Then we study the influence of mobility to obtain further results in subcritical secondary network. Finally, we take the propagation delay into consideration to reach more general conclusions. The major contributions of this paper are as follows:

- We investigate the scaling behavior of transmission delay in large scale ad hoc CR networks in both Boolean Model and Random Connection Model of continuum percolation theory.

- We present either exact value or the lower bound of the transmission delay to distance ratio $\gamma\left(\lambda_{S}, \mathcal{A}_{p}\right)$ as the distance goes to $\infty$. To the best of our knowledge, this is the first work that ever gives a precise description of delay's scaling behavior in CR networks.

- We do considerable amount of simulations to verify our theoretical analysis.

The rest of the paper is organized as follows. In section 2, we introduce our system model. In section 3, we present our main results on $\gamma\left(\lambda_{S}, \mathcal{A}_{p}\right)$ while ignoring the propagation delay. In sections 4 and 5 , we give detailed proof of our results. In section 6 , we take the propagation delay into consideration to obtain further results on $\gamma\left(\lambda_{S}, \mathcal{A}_{p}\right)$. Simulation results will be presented in section 7 to verify our theoretically analysis. Finally, we conclude in section 8 .

\section{SYSTEM MODEL}

We model the large scale $\mathrm{CR}$ ad hoc networks as a random geometric graph. Both primary users and secondary users are distributed according to the Poisson Point Process with density $\lambda_{P T}$ and $\lambda_{S}$, respectively, in an infinite two-dimensional space $\mathbb{R}^{2}$. Primary nodes, in other words spectrum owners, have higher priority over secondary nodes for using communication spectrum. Secondary users buy spectra and can use them while they are not used by primary users. It is clear that the waiting delay of primary network does not conclude the waiting time for spectrum opportunities. Then, the analysis of delay in primary network is a special case of that in secondary network. Therefore, we will focus on investigating the transmission delay of secondary network in the following parts. ${ }^{2}$

\subsection{The Primary Network Model}

We model the primary transmitters according to the Poisson Point Process $\mathbb{P}_{P T}(t)$ with density $\lambda_{P T}$. For one primary transmitter, its corresponding receivers are uniformly distributed within its transmission range $R_{p}$. Based on the displacement theorem in [3], we have that the primary receivers also form a two-dimensional Poisson Point Process $\mathbb{P}_{P R}(t)$. Note that the two Poisson processes are correlated with each other.

Assume that time is slotted with slot length $T_{S}$. Without lose of generality, we set $T_{S}=1$. Each primary transmitter is associated with an independent identically distributed (i.i.d.) switching renewal process, denoted by $S_{P}(t)$, which changes between two states: the active state, when it is broadcasting messages, and the sleep state, when it is inactive. A primary receiver is active or inactive identically to its corresponding transmitter. By the Markovian property of behaviors of primary network, the process $S_{p}(t)$ can be viewed as a discrete time Markov on-off (active-sleep) process with i.i.d. active periods $Q_{p}^{1}(t)$, and i.i.d. sleep periods $Q_{p}^{0}(t)$.

\footnotetext{
${ }^{2}$ In the following sections, we will use transmission delay of CR networks and transmission delay of secondary network interchangeably.
} 
Let us denote the probability of going from active state to sleep state and probability of going from sleep state to active state by $p_{10}$ and $p_{01}$, respectively. And the probability of sustaining active and sleep state in next slot are denoted by $p_{11}$ and $p_{00}$, respectively. Then $p_{01}=1 / E\left[Q_{p}^{0}(t)\right]$ and $p_{10}=1 / E\left[Q_{p}^{1}(t)\right]$. The stationary distribution of $S_{p}(t)$ is:

$$
\begin{aligned}
& \beta_{0} \triangleq \lim _{t \rightarrow \infty} \operatorname{Pr}\left(S_{P}(t)=0\right)=\frac{E\left[Q_{p}^{0}(t)\right]}{E\left[Q_{p}^{1}(t)\right]+E\left[Q_{p}^{1}(t)\right]},{ }^{3} \\
& \beta_{1} \triangleq \lim _{t \rightarrow \infty} \operatorname{Pr}\left(S_{P}(t)=1\right)=\frac{E\left[Q_{p}^{0}(t)\right]}{E\left[Q_{p}^{0}(t)\right]+E\left[Q_{p}^{1}(t)\right]} .
\end{aligned}
$$

The transmission range of primary users is $R_{p}$. Spectrum opportunities are not available for secondary network within a circle centered at one active primary user with radii $R_{p}$. Then the primary network at time slot $t$ can be represented by $\mathcal{A}_{P}\left(\lambda_{P T} ; S_{P}(t) ; R_{p}\right)$.

\subsection{The Secondary Network Model}

As we have discussed above, in [1] two models, i.e., Boolean Model (BM) and Random Connection Model (RCM), are used to describe the connectivity of a random geometric graph. If we model the connectivity of secondary network by BM, two secondary nodes share a link whenever they are within each other's transmission range. However, if connectivity of secondary users are modeled by RCM, we say two that secondary nodes share a link, with a positive probability, if and only if their distance is smaller than $r_{s}$. The positive probability is not one since the severe enemy attack, natural hazards, or energy depletion may cause link failure. In other words, such a positive probability indicates that the link is unreliable, which is the key difference between BM and RCM. Since $\mathrm{BM}$ and RCM are both non-trivial model, this paper will contain the results for both of them.

Two secondary users can exchange information only when they have at least one open path $\pi$. The following two conditions are necessary for the openness of $\pi$ :

1. $\pi$ is established through multiple links;

2. messages, broadcasted by the source node, can be forwarded by other nodes in $\pi$ to the destination node.

In RCM model, we will define a probability function $f(r)$ indicating the connection probability of two secondary nodes with distance $r$. In reality, the farther two nodes are apart, the higher risk for a successful communication connectivity. Moreover, when $r>r_{s}$, we assume there exists no link and thus $r_{s}$ can be regarded as secondary user's transmission range. Therefore, it is reasonable to assume that $f(r)$ is a monotone decreasing function with respect to $r$ and $f(r)=0$ whenever $r>r_{s}$. Formally, the constraint of $h(r)$ can be written as:

$$
0<f\left(r_{s}\right)<f(r)<f(0)<1,0<r<r_{s}{ }^{4}
$$

In $\mathrm{BM}$, two secondary nodes are connected only when they are within each other transmission range $r_{s}$. The probability function $h(r)$ is:

$$
h(r)=1,0<r \leq r_{s} .
$$

The problem of transmission delay will be discussed using both the BM and RCM in this article. We denote the BM and RCM modeled secondary network by $\mathcal{S}_{t}\left(\lambda_{S} ; h(r)\right)$ and $\mathcal{S}_{t}\left(\lambda_{S} ; f(r)\right)$, respectively, in each slot $t$. The subscript $t$ indicates that the network is

\footnotetext{
${ }^{3}$ In this paper, we use $\operatorname{Pr}(\cdot)$ to denote the probability of one certain event and $E[\cdot]$ denotes the expectation of one random variable.

${ }^{4} h(r)$ also satisfies $\int_{R^{2}} f(r) d r<\infty$ according to [1].
}

dynamic. Similarly, $\mathcal{G}_{t}\left(\lambda_{S} ; h(r) ; \mathcal{A}_{P}\right)$ and $\mathcal{G}_{t}\left(\lambda_{S} ; f(r) ; \mathcal{A}_{P}\right)$ denotes the CR networks modeled by BM and RCM, respectively. ${ }^{5}$

\subsection{Percolation of CR Networks}

Percolation theory, especially continuum percolation, is our major theoretical tool for investigating the transmission delay. Since we focus on delay problem in secondary network, we will use percolation of secondary network and percolation of CR networks interchangeably in the rest of the paper. Suppose at the beginning, time slot 0 , secondary nodes are distributed uniformly according to $\mathbb{P}_{S}(t)$ with density $\lambda_{S}$ in an infinite 2 -dimensional space with initial position $\mathfrak{X}^{0}=\left\{\mathcal{X}_{0}^{0}, \mathcal{X}_{1}^{0}, \ldots, \mathcal{X}_{n}^{0}, \ldots\right\}$. We denote the component containing the origin $\mathcal{O}$ by $\mathcal{C}_{\mathcal{O}}^{0}$ and the critical percolation probability by $\lambda_{c}(h(r))$. Note that if $\lambda_{S}>\lambda_{c}(h(r))$, the BM network $\mathcal{G}_{t}\left(\lambda_{S} ; h(r) ; \mathcal{A}_{P}\right)$ is percolated for all time slot $t$ and $\mathcal{C}_{\mathcal{O}}^{0}$ contains infinite nodes. Whereas if $\lambda_{S}<\lambda_{c}(h(r)), \mathcal{G}_{t}\left(\lambda_{S} ; h(r) ; \mathcal{A}_{P}\right)$ is not percolated for all $t$. Formally, we give the following definition of the critical density $\lambda_{c}(h(r))$ :

DEFINITION 1. For $\mathcal{G}_{t}\left(\lambda_{S} ; h(r) ; \mathcal{A}_{P}\right)$, the percolation probability $p_{\lambda}$ is the probability that the connected component containing the origin $\mathcal{C}_{\mathcal{O}}^{0}$ has infinite secondary users. The critical density of secondary users is defined as $\lambda_{c}(h(r))=\inf \left\{\lambda_{S}>0: p_{\lambda}>0\right\}$.

Similarly, the RCM network $\mathcal{G}_{t}\left(\lambda_{S} ; f(r) ; \mathcal{A}_{P}\right)$ is percolated if and only if $\lambda_{S}>\lambda_{c}(f(r))$. The critical density $\lambda_{c}(f(r))$ can be defined similarly to Definition 1 . Note that probability function $h(r)>f(r)$ for $\forall r \in\left[0, r_{s}\right]$, thus it is clear that the following lemma holds.

LEMMA 1. For the two critical percolation probabilities $\lambda_{c}(f(r))$ and $\lambda_{c}(h(r))$, they satisfy $\lambda_{c}(f(r))>\lambda_{c}(h(r))$.

We will further illustrate this lemma through simulation results in section 7 .

\subsection{Useful Notations}

To make it more readable, we list some useful notations and symbols used in this paper as follows:

- $\mathcal{A}_{P}$ : primary network. $\mathcal{G}_{t}\left(\lambda_{S} ; h(r) ; \mathcal{A}_{P}\right), \mathcal{G}_{t}\left(\lambda_{S} ; f(r) ; \mathcal{A}_{P}\right)$ : CR network modeled by BM and RCM, respectively.

- $\mathcal{D}(u, R)$ : a two-dimensional circular region centered at $u$ with radii $R . d(u, v)$ : the Eustachian distance between node $u$ and $v$;

- $S_{p}(t)$ : i.i.d. switching renewal process of each primary user. $p_{00}, p_{01}, p_{10}, p_{11}$ : four state switching probabilities of process $S_{p}(t)$;

- $H_{m}(t)$ : a discrete time Markov on-off (active-sleep) process with i.i.d. active periods $Z_{p}^{1}(t)$, and i.i.d. sleep periods $Z_{p}^{0}(t)$

- $\pi(u, v)$ : a path between node $u$ and $v$. $\mathcal{T}_{p}\left(l_{k}\right)$ : passing time of one link $l_{k} \in \pi(u, v)$. $\mathcal{I}\left(l_{k}\right)$ : the interfered region of $l_{k} . \mathcal{T}(\pi)$ : transmission delay of $\pi(u, v)$ in supercritical secondary network.

- $\mathcal{C}_{t, u, h(r)}$ : one connected cluster containing node $u$ at slot $t$ in subcritical secondary network. $\mathcal{M}\left\{\mathfrak{X}^{0}, d\right\}$ : the Constrained Circular I.I.D. Mobility Model for secondary network. $\mathcal{M}_{h(r)}\left(\lambda_{S}, \mathcal{A}_{P}\right)$ : a random variable characterized the physical size of one cluster.

${ }^{5}$ In following sections, we will use $\mathcal{A}_{P}$ to denote the primary network $\mathcal{A}_{P}\left(\lambda_{P T} ; S_{P}(t) ; R_{p}\right)$ for simplicity. 
- $\Upsilon$ : multi-cluster hop transmission process in a subcritical secondary network. $\mathcal{T}_{P}(\Upsilon)$ : cluster to cluster transmission delay of $\Upsilon . \mathcal{T}_{S}(\Upsilon)$ : waiting delay for spectrum opportunities in $\Upsilon$.

\section{MAIN RESULTS}

After introduction of the system model, we are ready to present our main results. Before that, we first give some basic properties of $\gamma\left(\lambda_{S}, \mathcal{A}_{P}\right)$ to make the subsequent analysis more smooth.

\subsection{Basic Properties of $\gamma\left(\lambda_{S}, \mathcal{A}_{P}\right)$}

$\gamma\left(\lambda_{S}, \mathcal{A}_{P}\right)$ is the ratio of transmission delay to distance as the distance from source to destination goes to $\infty$. We use the symbol $\gamma\left(\lambda_{S}, \mathcal{A}_{P}\right)$ to indicate that $\gamma$ is impacted by $\lambda_{S}$ and $\mathcal{A}_{P}$. First, $\lambda_{S}$ determines that whether the network contains a unique infinite connected component or only finite connected clusters. Second, $\mathcal{A}_{P}$ imposes interference on communication in secondary network because information can be forwarded by a secondary node only when it has spectrum opportunities.

W. Peng et al. [10] and W. Ren et al. [11] proved that percolation of supercritical secondary network still exists if the density of primary users $\lambda_{P T}$ is less than a critical number $\lambda_{P T}^{*}$. However, if $\lambda_{P T}>\lambda_{P T}^{*}$, although percolation phenomenon still exists topologically, the infinite connected component has broken into mutually disconnected finite clusters from the perspective of spectrum opportunities. In other words, although two disjoint clusters are connected topologically, they are not able to exchange messages due to lack of available communication channels. Therefore, to avoid confusion, it is necessary to redefine the supercritical and subcritical property of CR networks.

Definition 2. For $\mathcal{G}_{t}\left(\lambda_{S} ; h(r) ; \mathcal{A}_{P}\right)$,

1. If $\lambda_{S}<\lambda_{c}(h(r))$, the secondary network is subcritical.

2. If $\lambda_{S}>\lambda_{c}(h(r))$, the secondary network is supercritical. And if $\lambda_{P T}<\lambda_{P T}^{*}$, it is com-supercritical ("com" is short for "communication"). Whereas the secondary network is top-supercritical ("top" is shortfor "topology") when $\lambda_{P T}>$ $\lambda_{P T}^{*}$.

For $\mathcal{G}_{t}\left(\lambda_{S} ; f(r) ; \mathcal{A}_{P}\right)$, we have the similar definition. Note that if the secondary network is supercritical, the unique infinite connected component $\mathcal{C}_{\mathcal{O}}^{0}$ always exists from the perspective of topology structure and all nodes with within it share instantaneous connectivity. However, even two topologically connected nodes may not be able to communicate with each other if they lack spectrum opportunities. The difference between the two kinds of supercritical network is that all nodes within $\mathcal{C}_{\mathcal{O}}^{0}$ have spectrum opportunities in com-supercritical network, whereas a high fraction of nodes in $\mathcal{C}_{\mathcal{O}}^{0}$ do not have spectrum opportunities in top-supercritical network. Then we are ready to present the basic properties of $\gamma\left(\lambda_{S}, \mathcal{A}_{P}\right)$ in different categories of secondary network.

THEOREM 1. $\gamma\left(\lambda_{S}, \mathcal{A}_{P}\right)$ is characterized by the following basic properties:

1. $\gamma\left(\lambda_{S}, \mathcal{A}_{P}\right)$ increases with respect to $\lambda_{P T}$.

2. $\gamma\left(\lambda_{S}, \mathcal{A}_{P}\right)=0$ for any com-supercritical secondary network.

3. $\gamma\left(\lambda_{S}, \mathcal{A}_{P}\right)$ is a constant for a given top-supercritical secondary network.

4. $\gamma\left(\lambda_{S}, \mathcal{A}_{P}\right)$ has both lower and upper bound in subcritical secondary network.
We will present the detailed proof of these four items in the following sections.

\subsection{Main Results on $\gamma\left(\lambda_{S}, \mathcal{A}_{P}\right)$}

THEOREM 2. Given a BM Network $\mathcal{G}_{t}\left(\lambda_{S} ; h(r) ; \mathcal{A}_{P}\right)$ with $\lambda_{S}$ $>\lambda_{c}(h(r))$, consider two secondary users $u$ (source) and $v($ destination) connected by the path $\pi(u, v)$ in $\mathcal{C}_{\mathcal{O}}^{0}$, the corresponding $\gamma\left(\lambda_{S}, \mathcal{A}_{P}\right) \triangleq \lim _{d(u, v) \rightarrow \infty} \frac{\mathcal{T}(\pi)}{d(u, v)}$ satisfies:

(i) $\gamma\left(\lambda_{S}, \mathcal{A}_{P}\right)=0$ if $\lambda_{P T}<\lambda_{P T}^{*}\left(\lambda_{S}\right) ;{ }^{6}$

(ii) if $\lambda_{P T}>\lambda_{P T}^{*}\left(\lambda_{S}\right)$,

$$
\gamma\left(\lambda_{S}, \mathcal{A}_{P}\right)=\kappa \cdot \frac{1-\beta_{0}^{\bar{\psi}}}{\beta_{0}^{\bar{\psi}}},
$$

where $\kappa$ is a constant independent on $\lambda_{S} . \bar{\psi}$ is a constant satisfying $\left\lfloor\pi R_{p}^{2} \lambda_{P T}\right\rceil \leq \bar{\psi} \leq\left\lfloor\left[2 \pi R_{p}^{2}-\mathcal{F}\left(r_{s}, R_{p}\right)\right] \lambda_{P T}\right\rceil,{ }^{7}$ where $\mathcal{F}\left(r_{s}, R_{p}\right)=2 R_{p}^{2} \arccos \frac{r_{s}}{2 R_{p}}-\frac{r_{s} \sqrt{4 R_{p}^{2}-r_{s}^{2}}}{2}$.

THEOREM 3. For a RCM Network $\mathcal{G}_{t}\left(\lambda_{S} ; f(r) ; \mathcal{A}_{P}\right)$ with $\lambda_{S}$ $>\lambda_{c}(f(r))$, the conclusion on $\gamma\left(\lambda_{S}, \mathcal{A}_{P}\right)$ in Theorem 2 still holds.

THEOREM 4. For a BM Network $\mathcal{G}_{t}\left(\lambda_{S} ; h(r) ; \mathcal{A}_{P}\right)$ with density $\frac{\lambda_{c}(h(r))}{\mathcal{R}^{2}}<\lambda_{S}<\lambda_{c}(h(r))$, under the Constrained Circular I.I.D. Mobility Model $\mathcal{M}\left\{\mathfrak{X}^{0}, d\right\}$, the corresponding $\gamma\left(\lambda_{S}, \mathcal{A}_{P}\right) \triangleq$ $\lim _{d(u, v) \rightarrow \infty} \frac{\mathcal{T}_{P}(\Upsilon)+\mathcal{T}_{S}(\Upsilon)}{d(u, v)}$ satisfies $^{8}$ :

$$
\gamma\left(\lambda_{S}, \mathcal{A}_{P}\right) \geq \frac{1}{\beta_{0}^{\eta} \cdot E\left[\mathcal{M}_{h(r)}\left(\lambda_{S}, \mathcal{A}_{P}\right)+r_{s}\right]},
$$

where $\mathcal{R}=\frac{4 d+r_{s}}{r_{s}}$ and $\eta$ is a constant satisfying $\left\lfloor\pi R_{p}^{2} \lambda_{P T}\right\rceil<\eta$ $\leq\left\lfloor\pi\left(\frac{\mathcal{M}_{h(r)}\left(\lambda_{S}, \mathcal{A}_{P}\right)}{2}+R_{p}\right)^{2} \lambda_{P T}\right\rceil$.

\section{4. $\gamma\left(\lambda_{S}, \mathcal{A}_{P}\right)$ IN SUPERCRITICAL NETWORKS}

Given supercritical network, $\mathcal{C}_{\mathcal{O}}^{0}$ contains infinite secondary user$\mathrm{s}$ for all $t$. Two randomly chosen secondary users within $\mathcal{C}_{\mathcal{O}}^{0}$ share instantaneous connectivity and the transmission delay consists of only the waiting time for spectrum opportunities if we ignore propagation delay.

\section{1 $\gamma\left(\lambda_{S}, \mathcal{A}_{P}\right)$ for Supercritical $\mathcal{G}_{t}\left(\lambda_{S} ; h(r) ; \mathcal{A}_{P}\right)$}

If $\lambda_{P T}<\lambda_{P T}^{*}\left(\lambda_{S}\right)$, the network is com-supercritical. Then $(i)$ of Theorem 2 is clear since $\mathcal{T}(\pi)=0$.

If $\lambda_{P T}>\lambda_{P T}^{*}\left(\lambda_{S}\right)$, the network is top-supercritical. Now, given two secondary users $u$ (source) and $v$ (destination), an open path $\pi(u, v)$ in $\mathcal{C}_{\mathcal{O}}^{0}$ may be demolished when its node fails to forward the information to the destination due to the impact of primary network. Thus, $v$ can not receive the messages immediately and $\mathcal{T}(\pi)$ is no longer zero. Next, we will study $\gamma\left(\lambda_{S}, \mathcal{A}_{P}\right)$ according to the following computation flow.

\footnotetext{
${ }^{6}$ According to [11], we use the notation $\lambda_{P T}^{*}\left(\lambda_{S}\right)$ to indicate that the critical density $\lambda_{P T}^{*}$ varies with $\lambda_{S}$.

${ }^{7}\lfloor A\rceil$ denotes the largest integer less than or equal to $A$.

${ }^{8}$ Here, the expression of $\gamma\left(\lambda_{S}, \mathcal{A}_{P}\right)$ is different from that in Theorem 2 since $u$ and $v$ can not see any path connecting them when the network is subcritical. The messages broadcasted by $u$ is forwarded in multi-cluster hop transmission process, which will be introduced in section 5 .
} 


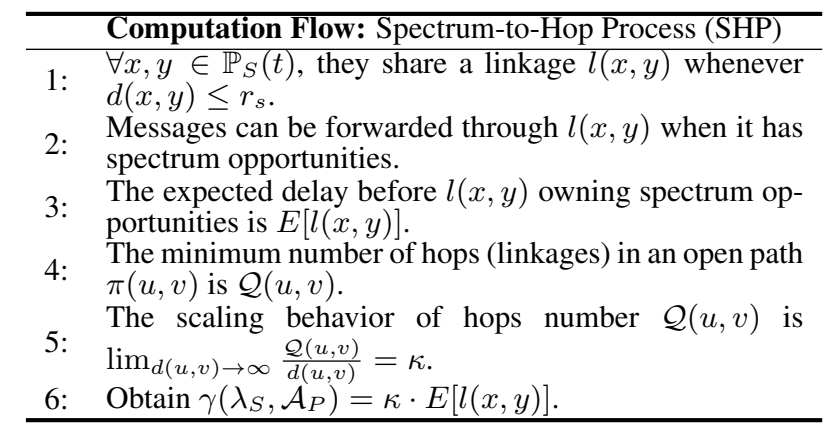

It is clear that after the step 1 in the above flow, the topological structure of $\mathcal{G}_{t}\left(\lambda_{S} ; h(r) ; \mathcal{A}_{P}\right)$ is established. When a BM modeled network is top-supercritical, we can find a topologically connected path $\pi(u, v)$ in $\mathcal{C}_{\mathcal{O}}^{0}$ after step 1 . For one link $l_{k}$ in $\pi(u, v)$, with two endpoints $u_{k}, u_{k+1}$ and $d\left(u_{k}, u_{k+1}\right)=r_{k}$, its passing time $\mathcal{T}_{p}\left(l_{k}\right)$ is zero when none of the active primary users have imposed interference on it. However, $\mathcal{T}_{p}\left(l_{k}\right)>0$ if $l_{k}$ is interfered by at least one active primary user. Therefore $\mathcal{T}_{p}\left(l_{k}\right)$ is delay before the messages can be forwarded through $l_{k}$.

For $l_{k}$, we can define its interfered region $\mathcal{I}\left(l_{k}\right)$. The transmission range of primary transmitters within this interfered region covers at least one of the endpoints of $l_{k}$. Hence, if any of these primary transmitters are active, no spectrum opportunities will be available for $u_{k}$ or $u_{k+1}$ and messages can not be forwarded through $l_{k}$ successfully. Formally, we give the definition of $\mathcal{I}\left(l_{k}\right)$ as follows.

DEFINITION 3. For any point $w$ in the same 2-dimensional $s$ pace as the secondary network, if $(i): d\left(w, u_{k}\right) \leq R_{p}$ or $(i i)$ : $d\left(w, u_{k+1}\right) \leq R_{p}$ or both $(i)$ and (ii) are satisfied, then we have $w \in \mathcal{I}\left(l_{k}\right)$.

Assume that $l_{k}$ is interfered by $\psi$ primary transmitters. By the Poisson Point Process $\mathbb{P}_{P T}(t)$ formed by primary transmitters with density $\lambda_{P T}$, we have the following lemma.

Lemma 2. (Step 2 in Flow SHP) Given the Poisson Point Process $\mathbb{P}_{P T}(t)$ formed by primary users with density $\lambda_{P T}$, for a link $l_{k}$, with length $r_{k} \leq r_{s}$ in $\pi(u, v)$, the expected number of primary transmitters $\psi$ within $\mathcal{I}\left(l_{k}\right)$ satisfies

$$
\begin{aligned}
& \text { 1. if } R_{p} \geq \frac{r_{k}}{2}, \\
& \qquad \psi=\left\lfloor\left[2 \pi R_{p}^{2}-\mathcal{F}\left(r_{k}, R_{p}\right)\right] \lambda_{P T}\right\rceil, \\
& \text { where } \mathcal{F}\left(r_{k}, R_{p}\right)=2 R_{p}^{2} \arccos \frac{r_{k}}{2 R_{p}}-\frac{r_{k} \sqrt{4 R_{p}^{2}-r_{k}^{2}}}{2} . \\
& \text { 2. if } R_{p}<\frac{r_{k}}{2} \\
& \qquad \psi=\left\lfloor 2 \pi R_{p}^{2} \lambda_{P T}\right\rceil .
\end{aligned}
$$

PROOF. If $R_{p} \geq \frac{r_{k}}{2}$, illustrated in Figure $1 \mathrm{a}$, two circular region $\mathcal{D}\left(u_{k}, R_{p}\right)$ and $\mathcal{D}\left(u_{k+1}, R_{p}\right)$ are intersected with each other. According to Definition 3,

$$
\mathcal{I}\left(l_{k}\right)=\mathcal{D}\left(u_{k}, R_{p}\right) \bigcup \mathcal{D}\left(u_{k+1}, R_{p}\right)
$$

Considering $\mathbb{P}_{P T}(t)$ with density $\lambda_{P T}$, the expected number of primary transmitters $\psi$ within $\mathcal{I}\left(l_{k}\right)$ is

$$
\psi=\left\lfloor\lambda_{P T} \cdot\left|\mathcal{I}\left(l_{k}\right)\right|\right\rceil,
$$

where $\left|\mathcal{I}\left(l_{k}\right)\right|$ denotes the Lebesgue measure (or area) of $\mathcal{I}\left(l_{k}\right)$. By tools in plane geometry, we have

$$
\left|\mathcal{I}\left(l_{k}\right)\right|=2 \pi R_{p}^{2}-\mathcal{F}\left(r_{k}, R_{p}\right)
$$

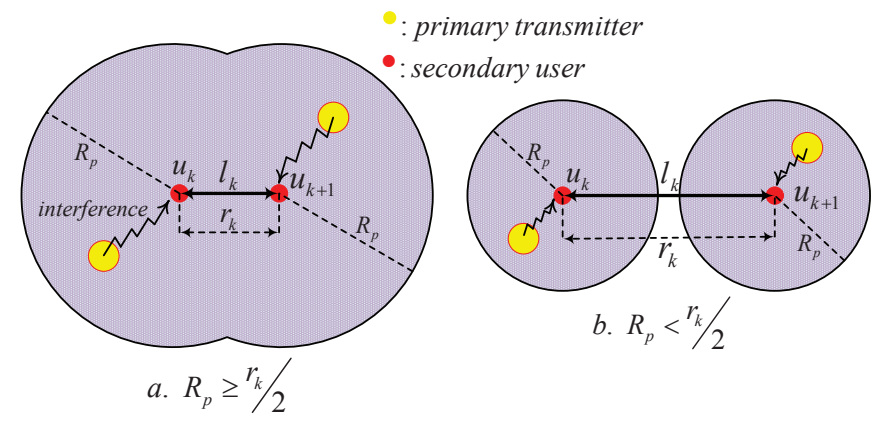

Figure 1: Illustration of Lemma 2.

where $\mathcal{F}\left(r_{k}, R_{p}\right)=2 R_{p}^{2} \arccos \frac{r_{k}}{2 R_{p}}-\frac{r_{k} \sqrt{4 R_{p}^{2}-r_{k}^{2}}}{2}$. Combining Equations (2) and (3), item 1 is proved.

If $R_{p}<\frac{r_{k}}{2}, \mathcal{D}\left(u_{k}, R_{p}\right)$ and $\mathcal{D}\left(u_{k+1}, R_{p}\right)$ are two independent areas and the expected number of primary transmitters in the two areas are also independent. Then item 2 can be proved by simply by substituting $\left|\mathcal{I}\left(l_{k}\right)\right|=2 \pi R_{p}^{2}$ into Equation (2).

Note that in practical networks, $R_{p}$ is larger than $r_{s}$ since the transmission power of primary transmitter may be several times larger than that of secondary user. To avoid the triviality in our analysis, we assume that $R_{p}>r_{s}$. Then the first item of Lemma 2 is always satisfied since $r_{s} \geq r_{k}$. According to Equation (1), it is clear that $\psi$ depends on $r_{k}$. However, due to homogeneity of the Poisson Point Process and Boolean Model, we can use the expectation of $E[\psi]$ to approximate $\psi$ since $\psi$ converges to $E[\psi]$ when the subscript $k$ goes to $\infty$. By $0<r_{k} \leq r_{s}$, we have

$$
\left\lfloor\pi R_{p}^{2} \lambda_{P T}\right\rceil \leq E[\psi] \leq\left\lfloor\left[2 \pi R_{p}^{2}-\mathcal{F}\left(r_{s}, R_{p}\right)\right] \lambda_{P T}\right\rceil .
$$

Thus $E[\psi]$ is a constant, independent on $k$, between the lower and upper bound in Equation (4). For simplicity, we will use $\bar{\psi}$ to denote $E[\psi]$ in the rest of paper.

We associate one random variable $H_{m}(t)$ for each link $l_{m} \in$ $\pi(u, v)$. For $l_{k}, H_{k}(t)=0$ only when all the primary transmitters in $\mathcal{I}\left(l_{k}\right)$ are sleep and $H_{k}(t)=1$ if at least one of these primary transmitters is active. By the Markovian property behaviors of each primary user, the process $H_{m}(t)$ can be viewed as a discrete time Markov on-off (active-sleep) process with i.i.d. active periods $Z_{p}^{1}(t)$, and i.i.d. sleep periods $Z_{p}^{0}(t)$. Thus, $\mathcal{T}_{p}\left(l_{k}\right)$ is the delay before $H_{k}(t)=0$ for the first time, i.e.,

$$
\mathcal{T}_{p}\left(l_{k}\right)=\inf _{t \geq 0}\left\{t: H_{k}(t)=0\right\} .
$$

The expected delay for $l_{k}$ (step 3 in Flow SHP) is:

$$
E\left[\mathcal{T}_{p}\left(l_{k}\right)\right]=\sum_{n=0}^{\infty} n\left(1-\beta_{0}^{\bar{\psi}}\right)^{n} \cdot \beta_{0}^{\bar{\psi}}=\frac{1-\beta_{0}^{\bar{\psi}}}{\beta_{0}^{\bar{\psi}}} .
$$

Now we have obtained the expected delay for each hop in $\pi(u, v)$. Next we will investigate the scaling behavior of the number of hops in the path.

LEMMA 3. (Steps 4 and 5 in Flow SHP) Given the top-supercritical Boolean Model Network $\mathcal{G}_{t}\left(\lambda_{S} ; h(r) ; \mathcal{A}_{P}\right)$, the minimum number of hops $\mathcal{Q}(u, v)$ in one path $\pi(u, v)$ scales linearly with the distance $d(u, v)$, i.e.,

$$
\lim _{d(u, v) \rightarrow \infty} \frac{\mathcal{Q}(u, v)}{d(u, v)}=\kappa .
$$


PROOF. The proof of lemma 3 uses Liggett's subadditive ergodic theorem [2]. This technique has already been explored in [13] and [14]. Here we will omit the detailed proof because of the space limitation.

Combining Equation (5) and Lemma 3, we will finish the Computation Flow SHP after the step 6.

$$
\begin{aligned}
\gamma\left(\lambda_{S}, \mathcal{A}_{P}\right)= & \lim _{d(u, v) \rightarrow \infty} \frac{\mathcal{T}(\pi)}{d(u, v)}=\lim _{d(u, v), k \rightarrow \infty} \frac{\sum \mathcal{T}_{p}\left(l_{k}\right)}{d(u, v)} \\
= & \lim _{d(u, v) \rightarrow \infty} \frac{\mathcal{Q}(u, v) \cdot E\left[\mathcal{T}_{p}\left(l_{k}\right)\right]}{d(u, v)} \\
& (\text { the i.i.d. random variable sequence } \\
& \left.\sum \mathcal{T}_{p}\left(l_{k}\right) \text { converges to its expectation }\right) \\
= & \kappa \cdot \frac{1-\beta_{0}^{\bar{\psi}}}{\beta_{0}^{\bar{\psi}}} .
\end{aligned}
$$

Then we have proved the Theorem 2.

\section{2 $\gamma\left(\lambda_{S}, \mathcal{A}_{P}\right)$ for Supercritical $\mathcal{G}_{t}\left(\lambda_{S} ; f(r) ; \mathcal{A}_{P}\right)$}

To show the non-triviality of Theorem 3 , we need to introduce a algorithm named Removing and Extracting Process (REP) to reveal the relation between $\mathcal{G}_{t}\left(\lambda_{S} ; f(r) ; \mathcal{A}_{P}\right)$ and $\mathcal{G}_{t}\left(\lambda_{S} ; h(r) ; \mathcal{A}_{P}\right)$. According to our system model, it is clear that the primary network of the two CR networks are the same. So we can simply study the correlation between $\mathcal{S}_{t}\left(\lambda_{S} ; h(r)\right)$ and $\mathcal{S}_{t}\left(\lambda_{S} ; f(r)\right)$.

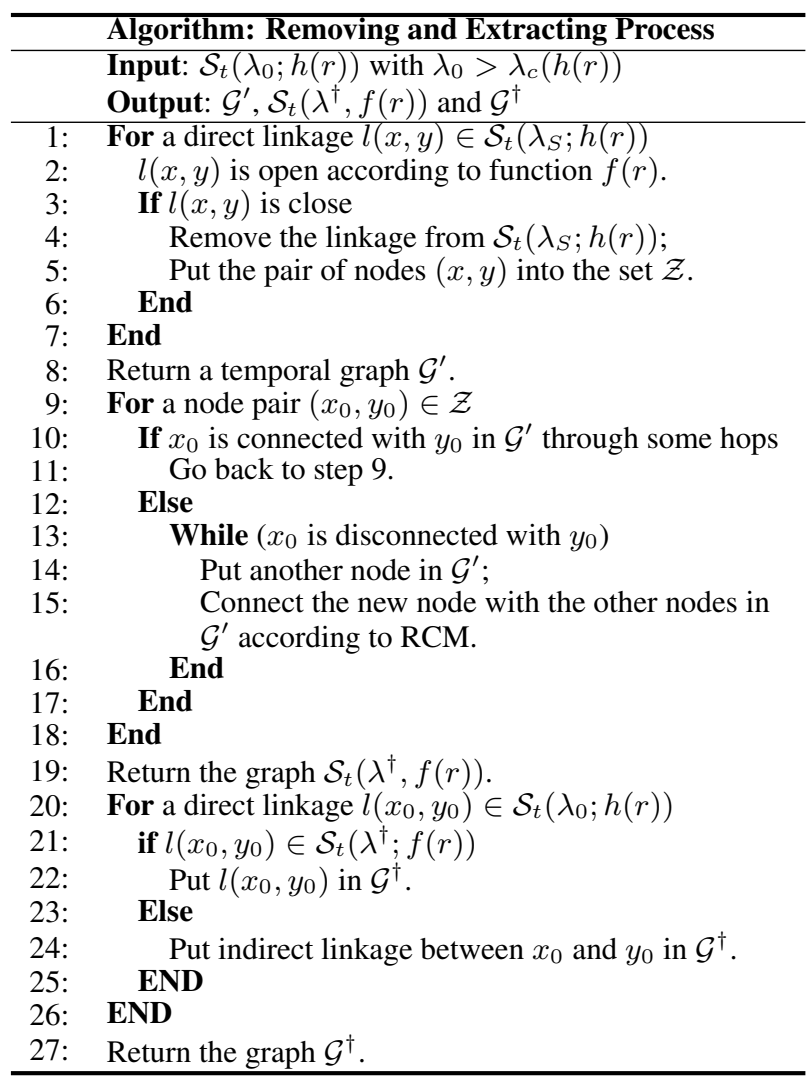

Steps 1 to 8 are the removing process and we get a temporal graph $\mathcal{G}^{\prime}$. It is clear that $\mathcal{G}^{\prime}=\mathcal{S}_{t}\left(\lambda_{0} ; f(r)\right)$ because we have reconstructed $\mathcal{S}_{t}\left(\lambda_{0} ; h(r)\right)$ according to the probability function $f(r)$. However, $\mathcal{G}^{\prime}$ may not be top-supercritical since $\lambda_{c}(h(r))<$ $\lambda_{c}(f(r))$ according to Lemma 1 . The purpose of steps 9 to 19 is to construct a top-supercritical network $\mathcal{S}_{t}\left(\lambda^{\dagger} ; f(r)\right)$. Steps 10 and

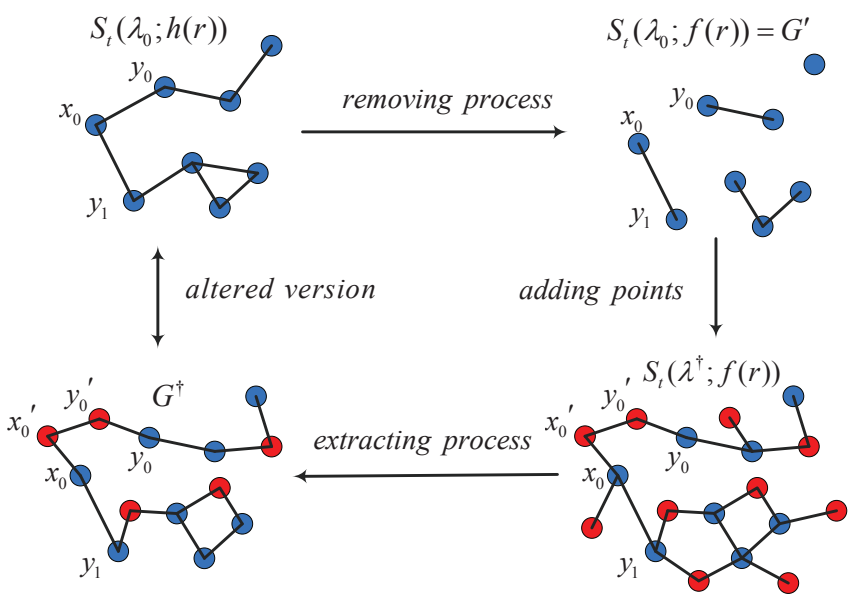

Figure 2: Illustration of Algorithm REP.

11 show a special case that even the direct linkage between $x_{0}$ and $y_{0}$ is removed, they are still connected through some hops. When $x_{0}$ and $y_{0}$ are disconnected, we need to put some new nodes into $\mathcal{G}^{\prime}$ through steps 12 to 17 . These new nodes are connected with each other and with the previous nodes in $\mathcal{G}^{\prime}$ according to RCM. Thus, when the node density is sufficiently large, i.e., the network is supercritical, we can find a newly established path between $x_{0}$ and $y_{0}$ with probability one. In steps 20 to 26 , we execute the extracting process to get a new graph $\mathcal{G}^{\dagger}$. The process is illustrated in Figure 2.

The purpose of Algorithm REP is to show that the new graph $\mathcal{G}^{\dagger}$ is an altered version of $\mathcal{S}_{t}\left(\lambda_{0} ; h(r)\right)$. Formally, we can write this in the following Lemma:

LEMMA 4. $\mathcal{G}^{\dagger}$ is an altered version of $\mathcal{S}_{t}\left(\lambda_{0} ; h(r)\right)$. Specifical$l y, \forall l(x, y) \in \mathcal{S}_{t}\left(\lambda_{0} ; h(r)\right), x$ and $y$ are connected either through a direct linkage $l(x, y)$ or through an indirect linkage ${ }^{9}$ in $G^{\dagger}$.

Proof. In Figure $2, l\left(x_{0}, y_{1}\right)$ is kept in both $\mathcal{S}_{t}\left(\lambda_{0} ; h(r)\right)$ and $\mathcal{S}_{t}\left(\lambda^{\dagger} ; f(r)\right)$. But $l\left(x_{0}, y_{0}\right)$ does not exist in $\mathcal{S}_{t}\left(\lambda^{\dagger} ; f(r)\right)$ and $x_{0}$ is connected to $y_{0}$ through an indirect linkage between $x_{0}$ and $y_{0}$. Therefore, if we extract all these nodes and linkages (direct and indirect ones) in $\mathcal{S}_{t}\left(\lambda^{\dagger} ; f(r)\right)$, we will get a new graph $\mathcal{G}^{\dagger}$. The only difference between $\mathcal{G}^{\dagger}$ and $\mathcal{S}_{t}\left(\lambda_{0} ; h(r)\right)$ is that a direct linkage $l(x, y) \in \mathcal{S}_{t}\left(\lambda_{0} ; h(r)\right)$ may be an indirect linkage in $\mathcal{G}^{\dagger}$. Thus, we can define $\mathcal{G}^{\dagger}$ as an altered version of $\mathcal{S}_{t}\left(\lambda_{0} ; h(r)\right)$.

Therefore, if we can find a path $\pi(u, v)$ from source $u$ to destination $v$ in $\mathcal{S}_{t}\left(\lambda_{0} ; h(r)\right)$ or $\mathcal{G}_{t}\left(\lambda_{S} ; h(r) ; \mathcal{A}_{P}\right)$, we can also find a path $\pi^{\prime}(u, v)$ in $\mathcal{G}^{\dagger}$. And the difference between $\pi^{\prime}(u, v)$ and $\pi(u, v)$ is that $\pi^{\prime}(u, v)$ might have more hops than $\pi(u, v)$. Fortunately, this does not make any difference when we consider their scaling behavior as only finite number of hops have been added in $\pi^{\prime}(u, v)$. Thus, we can conclude that Lemma 3 still holds for $\mathcal{G}^{\dagger}$. Since $\mathcal{G}^{\dagger}$ is a subgraph of $\mathcal{S}_{t}\left(\lambda^{\dagger} ; f(r)\right)$, Lemma 3 also holds for $\mathcal{S}_{t}\left(\lambda^{\dagger} ; f(r)\right)$. Formally, we can write this conclusion as follows:

LEMMA 5. Given the top-supercritical Random Connection Model Network $\mathcal{G}_{t}\left(\lambda_{S} ; f(r) ; \mathcal{A}_{P}\right)$, Lemma 3 still holds.

\footnotetext{
${ }^{9}$ An indirect linkage between $x_{0}$ and $y_{0}$ includes $x_{0}, y_{0}$ and the other nodes that are used to connected $x_{0}$ to $y_{0}$. For instance, the indirect linkage between $x_{0}$ and $y_{0}$ in $\mathcal{G}^{\dagger}$, illustrated in Figure 2, consists of $x_{0}, y_{0}, x_{0}^{\prime}, y_{0}^{\prime}$ and their linkages.
} 
Our simulation results also verify this lemma. Then the proof of Theorem 3 is finished.

\section{5. $\gamma\left(\lambda_{S}, \mathcal{A}_{P}\right)$ IN SUBCRITICAL NETWORKS}

When it is subcritical, secondary network is broken into mutually disjoint finite clusters. Then the messages can not reach destination node if it is in different clusters with source node and the network's connectivity is fixed over time.

However, when the secondary network is mobile, there is a probability that the messages can be forwarded to destination in subcritical network. For instance, a source node broadcasts a message in a subcritical network at time 0 . Ignoring propagation delay, all nodes in the same cluster with the source node will receive the message instantaneously. The nodes in this cluster are only a fraction of all the nodes since the network is not percolated. As time goes on, however, nodes move, and there is a probability that the message can pass from one of these message-carrying nodes in the cluster to a new node if they are within each other's communication range. Then all other nodes in the same cluster with the newly informed node will also receive the information. As this process goes on, a large proportion of nodes will be informed of the message after some delay. In other words, the messages can spread and reach the destination in subcritical network if it is mobile. Thus, we need to introduce a mobility model for secondary network.

\subsection{Mobility Model}

We model the mobility of each secondary user by the constrained circular i.i.d. mobility model, which has been introduced in [13]. Formally, we give the definition of mobility model as follows:

DEFINITION 4. The Constrained Circular I.I.D. Mobility Model $\mathcal{M}\left\{\mathfrak{X}^{0}, d\right\}$ : Given the initial position of secondary nodes $\mathfrak{X}^{0}=$ $\left\{\mathcal{X}_{0}^{0}, \mathcal{X}_{1}^{0}, \ldots, \mathcal{X}_{n}^{0}, \ldots\right\}$, at each time slot $k=1,2,3, \ldots, \mathcal{X}_{u}^{k}$ is uniformly distributed randomly within $\mathcal{D}\left(\mathcal{X}_{u}^{k-1}, d\right)$ : a circular region centered at $\mathcal{X}_{u}^{k-1}$ with radii $d$. The positions $\mathcal{X}_{u}^{k}$ are mutually independent among all secondary users and independent of all previous positions $\mathcal{X}_{u}^{t^{\prime}}$ where $t^{\prime}=\{0,1, \ldots, k-1\} .{ }^{10}$

Note Theorems 2 and 3 still hold if we apply the mobility model in supercritical secondary network since mobility does not impact the percolation phenomenon in the network. However, in subcritical secondary network, mobility increase the probability of percolation. Specifically, two nodes with initial distance less than $r_{s}+4 d$ has a strictly positive probability that they can share link after finite delay. This indicates that the largest possible transmission range of each node is $r_{s}+4 d$ under the mobility model. Thus, the percolation property of the network under mobility model is the same as that of the static network in which secondary user's transmission range is $r_{s}+4 d$. According to the scaling property of BM in [1], we have the following lemma.

LEMMA 6. Given network $\mathcal{G}_{t}\left(\lambda_{S} ; h(r) ; \mathcal{A}_{p}\right)$ under the mobility model $\mathcal{M}\left\{\mathfrak{X}^{0}, d\right\}$, if we scale up ${ }^{11}$ the network by ratio $\mathcal{R}=$ $\frac{r_{s}+4 d}{r_{s}}$, the critical density for percolation after scaling is $\frac{\lambda_{c}(h(r))}{\mathcal{R}^{2}}$.

For a subcritical network with density $\lambda<\lambda_{c}(h(r))$, according to Lemma 6 , if $\lambda>\frac{\lambda_{c}(h(r))}{\mathcal{R}^{2}}$, the network still percolates. In other words, the weaker version of overall connectivity may be still established in a subcritical network.

\footnotetext{
${ }^{10}$ Note in our mobility model, the time it takes for a node to move to new position is not accounted. Thus, delay is independent on the mobility speed.

${ }^{11}$ Here, scaling up the network means increasing secondary user's transmission range while keeping their positions the same.
}

Up to now, we have showed that under Constrained Circular I.I.D. Mobility Model, information can spread among a subcritical network. However, the dissemination process is quite different from that in supercritical network. It is done through the process that we have briefly explained at the beginning of this section. We define this process as multi-cluster hop transmission process. To study the transmission delay, we first need to investigate this multicluster hop transmission process in the following subsection.

\subsection{Multi-Cluster Hop Transmission Process}

Given a cluster containing the secondary node $u_{0}$, denoted by $\mathcal{C}_{t, u_{0}, h(r)}$, all secondary nodes in it share instantaneous connectivity with $u_{0}$. We assume that the source node $u$ is in the same cluster with $u_{0}$. At the beginning, time slot $t_{0}=0$, all secondary nodes within $\mathcal{C}_{t_{0}, u_{0}, h(r)}$ are informed of the message, broadcasted by $u$, instantaneously since we have ignored the propagation delay. However, the transmission process stops within $\mathcal{C}_{t_{0}, u_{0}, h(r)}$ because no other clusters are connected with it. This transmission process will restart at time slot $t_{1}>t_{0}$, when at least one node $u_{1}$ is able to receive the information from $\mathcal{C}_{t_{0}, u_{0}, h(r)}$ and then all nodes in cluster $\mathcal{C}_{t_{1}, u_{1}, h(r)}$ will instantaneously informed of the messages. This process goes on, until at time slot $t_{M}$, node $u_{M}$ receives the information and the destination node $v$ is in the same cluster with $u_{M}$. Therefore, $v$ is informed of the information instantaneously at this time slot. Then messages have been transmitted from source node $u$ to destination node $v$ after this process. We name this process as multi-cluster hop transmission process, denoted by $\Upsilon\left\{\mathcal{C}_{t_{0}, u_{0}, h(r)}, \mathcal{C}_{t_{1}, u_{1}, h(r)}, \ldots, \mathcal{C}_{t_{M}, u_{M}, h(r)}\right\}$.

We associate each cluster $\mathcal{C}_{t_{k}, u_{k}}, h(r)$ with one random variable $\mathcal{M}_{t_{k}, u_{k}, h(r)}\left(\lambda_{S}, \mathcal{A}_{P}\right)$, which is defined as:

DEFINITION 5. For one cluster $\mathcal{C}_{t_{k}, u_{k}, h(r)}$,

$$
\mathcal{M}_{t_{k}, u_{k}, h(r)}\left(\lambda_{S}, \mathcal{A}_{P}\right)=\sup _{u_{k}^{\prime}, v_{k}^{\prime} \in \mathcal{C}_{t, u, h(r)}}\left\{d\left(u_{k}^{\prime}, v_{k}^{\prime}\right)\right\} .
$$

From the definition, it is clear that $\mathcal{M}_{t_{k}, u_{k}, h(r)}\left(\lambda_{S}, \mathcal{A}_{P}\right)$ is the

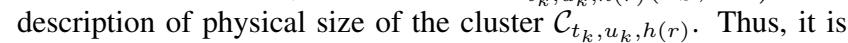
true that $\mathcal{M}_{h(r)}\left(\lambda_{S}, \mathcal{A}_{P}\right)$ increases with respect to $\lambda_{S}$ and decreases as the interference from primary network is heavier. Besides, $\mathcal{M}_{t_{k}, u_{k}, h(r)}\left(\lambda_{S}, \mathcal{A}_{P}\right)$ is independent on $u_{k}$ and $t_{k}$. So we will rewrite it as $\mathcal{M}_{h(r)}\left(\lambda_{S}, \mathcal{A}_{P}\right)$ for simplicity.

\section{$5.3 \gamma\left(\lambda_{S}, \mathcal{A}_{P}\right)$ for Subcritical Networks}

When $\mathcal{G}_{t}\left(\lambda_{S} ; h(r) ; \mathcal{A}_{P}\right)$ is subcritical, the messages must be forwarded in multi-cluster hop transmission process. Now given the source $u$ and destination $v$, consider the process $\Upsilon$ stating with $\mathcal{C}_{t_{0}, u_{0}, h(r)}$ and ending with $\mathcal{C}_{t_{M}, u_{M}, h(r)}$, as illustrated in Figure 3. Once we can find one cluster containing the $v$ receives the messages as $\Upsilon$ goes on, $v$ will be successfully informed of the information broadcasted by $u$. For one cluster $\mathcal{C}_{t_{m}, u_{m}, h(r)}(1 \leq m<M)$ in $\Upsilon$, it will be able to forward the messages to the next cluster $\mathcal{C}_{t_{m+1}, u_{m+1}, h(r)}$ only when both of the following two conditions are satisfied.

(i) For $v_{m} \in \mathcal{C}_{t_{m}, u_{m}, h(r)}, v_{m+1} \in \mathcal{C}_{t_{m+1}, u_{m+1}, h(r)}$

$$
d_{\text {min }}=\inf \left\{d\left(v_{m}, v_{m+1}\right)\right\}<r_{s}
$$

(ii) For the link $l_{v_{m}, v_{m+1}}$ in condition $(i)$, no active primary users are residing within the interfered region $\mathcal{I}\left(l_{v_{m}, v_{m+1}}\right)$ given in Definition 3.

Therefore, the total transmission delay $\mathcal{T}_{C}^{m}(\Upsilon)$ in the $m^{\prime}$ th hop $\mathcal{C}_{t_{m}, u_{m}, h(r)}$ of $\Upsilon$ are composed by cluster to cluster transmission delay (condition $i$ ) and the waiting delay for spectrum opportunities (condition $i$ ), denoted by $\mathcal{T}_{P}^{m}(\Upsilon)$ and $\mathcal{T}_{S}^{m}(\Upsilon)$, respectively. We 
The starting cluster The second cluster The Mth cluster

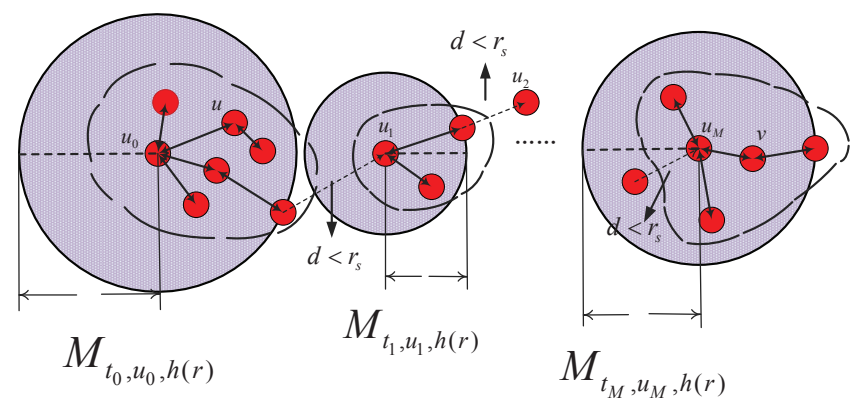

Figure 3: Illustration of the multi-cluster hop transmission process $\Upsilon$.

assume that the expected number of primary transmitters impacting $\mathcal{C}_{t_{m}, u_{m}, h(r)}$ is $\eta$. Using the same technique in Lemma 2, we can see $\eta$ is a constant satisfying:

$$
\left\lfloor\pi R_{p}^{2} \lambda_{P T}\right\rceil<\eta \leq\left\lfloor\pi\left(\frac{\mathcal{M}_{h(r)}\left(\lambda_{S}, \mathcal{A}_{P}\right)}{2}+R_{p}\right)^{2} \lambda_{P T}\right\rceil .
$$

Similar as the previous section, we use process $H_{m}(t)$ to denote the state transition process of these $\eta$ primary users. Then we have

$$
\begin{aligned}
\mathcal{T}_{C}^{m}(\Upsilon) & =\mathcal{T}_{P}^{m}(\Upsilon)+\mathcal{T}_{S}^{m}(\Upsilon) \\
& =\mathcal{T}_{P}^{m}(\Upsilon)+\inf _{t \geq \mathcal{T}_{P}^{m}(\Upsilon)}\left\{H_{m}(t)=0\right\}
\end{aligned}
$$

For $\mathcal{T}_{P}(\Upsilon)$, we have

$$
\begin{aligned}
\mathcal{T}_{P}(\Upsilon) & =\sum_{m=0}^{M-1} \mathcal{T}_{P}^{m}(\Upsilon)=\sum_{m=0}^{M-1}\left(t_{m+1}-t_{m}\right) \\
& =t_{M}-t_{0} \geq M
\end{aligned}
$$

Note that for $\forall m=0,1,2, \ldots, M-1, u_{m+1}$ is connected with one node $w_{m}$ in $\mathcal{C}_{t_{m}, u_{m}, h(r)}$ at slot $t_{m+1}$. Then their Euclidean distance satisfies the following inequality according to the triangle inequity:

$$
\begin{aligned}
\left\|u_{m+1}-u_{m}\right\| & \leq\left\|u_{m}-w_{m}\right\|+\left\|u_{m+1}-w_{m}\right\| \\
& \leq \mathcal{M}_{h(r)}\left(\lambda_{S}, \mathcal{A}_{P}\right)+r_{s} .
\end{aligned}
$$

By iteration of the inequalities as $\Upsilon$ goes on, the distance between $u$ and $v d(u, v)$ satisfies:

$$
\begin{aligned}
d(u, v) \leq & \sum_{m=0}^{M-1}\left\|u_{m+1}-u_{m}\right\|+\left\|u_{M}-v\right\| \\
\leq & \sum_{m=0}^{M-1}\left(\mathcal{M}_{h(r)}\left(\lambda_{S}, \mathcal{A}_{P}\right)+r_{s}\right)+\mathcal{M}_{h(r)}\left(\lambda_{S}, \mathcal{A}_{P}\right) \\
& \left(\text { since } u_{M} \text { and } v \text { are within one cluster }\right) \\
= & \sum_{m=0}^{M}\left(\mathcal{M}_{h(r)}\left(\lambda_{S}, \mathcal{A}_{P}\right)+r_{s}\right) .
\end{aligned}
$$

Moreover, according to the spatial independence of Poisson Point Process, $\mathcal{M}_{h(r)}\left(\lambda_{S}, \mathcal{A}_{P}\right)$ is an i.i.d. random variable. As $d(u, v)$ goes to $\infty$, the number of clusters $M$ in $\Upsilon$ also goes to $\infty$. Then we have

$$
\begin{aligned}
\frac{\mathcal{T}_{P}(\Upsilon)}{d(u, v)} \geq & \lim _{M \rightarrow \infty} \frac{M}{\sum_{m=0}^{M}\left(\mathcal{M}_{h(r)}\left(\lambda_{S}, \mathcal{A}_{P}\right)+r_{s}\right)} \\
& \left(\text { since } d(u, v) \leq \sum_{m=0}^{M}\left(\mathcal{M}_{h(r)}\left(\lambda_{S}, \mathcal{A}_{P}\right)+r_{s}\right)\right. \\
& \text { and } \left.\mathcal{T}_{P}(\Upsilon) \geq M\right) \\
= & \frac{1}{E\left[\mathcal{M}_{h(r)}\left(\lambda_{S}, \mathcal{A}_{P}\right)+r_{s}\right]} \\
& \quad \text { the average of an i.i.d. random variable } \\
& \text { sequence converges to its expectation). }
\end{aligned}
$$

$$
\begin{aligned}
\frac{\mathcal{T}_{S}(\Upsilon)}{d(u, v)} \geq & \lim _{M \rightarrow \infty} \sum_{m=0}^{M} \frac{\mathcal{T}_{S}^{m}(\Upsilon)}{\left(\mathcal{M}_{h(r)} \lambda_{S}, \mathcal{A}_{P}\right)+r_{s}} \\
& \left(\text { since } d(u, v) \leq \sum_{k=0}^{M}\left(\mathcal{M}_{h(r)}\left(\lambda_{S}, \mathcal{A}_{P}\right)+r_{s}\right)\right) \\
= & \lim _{M \rightarrow \infty} \sum_{m=0}^{M} \frac{\mathcal{T}_{S}^{m}(\Upsilon) / M}{\left(\mathcal{M}_{h(r)}\left(\lambda_{S}, \mathcal{A}_{P}\right)+r_{s}\right) / M} \\
= & \frac{E\left[\mathcal{T}_{S}^{m}(\Upsilon)\right]}{E\left[\mathcal{M}_{h(r)}\left(\lambda_{S}, \mathcal{A}_{P}\right)+r_{s}\right]} \\
= & \frac{1-\beta_{0}^{\eta}}{\beta_{0}^{\eta} \cdot E\left[\mathcal{M}_{h(r)}\left(\lambda_{S}, \mathcal{A}_{P}\right)+r_{s}\right]} .
\end{aligned}
$$

Summing up $\frac{\mathcal{T}_{P}(\Upsilon)}{d(u, v)}$ and $\frac{\mathcal{T}_{S}(\Upsilon)}{d(u, v)}$, we can obtain

$$
\begin{aligned}
\gamma\left(\lambda_{S}, \mathcal{A}_{P}\right) & =\frac{\mathcal{T}_{P}(\Upsilon)+\mathcal{T}_{S}(\Upsilon)}{d(u, v)} \\
& \geq \frac{1}{E\left[\mathcal{M}_{h(r)}\left(\lambda_{S}, \mathcal{A}_{P}\right)+r_{s}\right]} \\
& +\frac{1-\beta_{0}^{\eta}}{\beta_{0}^{\eta} \cdot E\left[\mathcal{M}_{h(r)}\left(\lambda_{S}, \mathcal{A}_{P}\right)+r_{s}\right]} \\
& =\frac{1}{\beta_{0}^{\eta} \cdot E\left[\mathcal{M}_{h(r)}\left(\lambda_{S}, \mathcal{A}_{P}\right)+r_{s}\right]}
\end{aligned}
$$

Then the proof of Theorem 4 is finished. However, we fail to give a tight upper bound of $\gamma\left(\lambda_{S}, \mathcal{A}_{P}\right)$ in subcritical network. But intuitively $\gamma\left(\lambda_{S}, \mathcal{A}_{P}\right)$ is not $\infty$.

\section{IMPACT OF PROPAGATION DELAY ON $\gamma\left(\lambda_{S}, \mathcal{A}_{P}\right)$}

In previous sections, while studying the $\gamma\left(\lambda_{S}, \mathcal{A}_{P}\right)$, we ignored the propagation delay. However, propagation delay may become dominant factor in some cases especially when the load of networks is heavy. In this section, we will consider the impact of propagation delay on $\gamma\left(\lambda_{S}, \mathcal{A}_{P}\right)$. For ease of analysis, we assume that the propagation delays $\tau$ is the same for different links. Moreover, we assume that $\tau<1$ since propagation delay is relatively small compared to the length of one time slot. Then we present our results on $\gamma\left(\lambda_{S}, \mathcal{A}_{P}\right)$ as follows:

THEOREM 5. Given a BM Network $\mathcal{G}_{t}\left(\lambda_{S} ; h(r) ; \mathcal{A}_{P}\right)$ with $n$ ode density $\lambda_{S}>\lambda_{c}(h(r))$, its $\gamma\left(\lambda_{S}, \mathcal{A}_{P}\right)$ satisfies:

(i) $\gamma\left(\lambda_{S}, \mathcal{A}_{P}\right)=\kappa \tau$ if $\lambda_{P T}<\lambda_{P T}^{*}\left(\lambda_{S}\right)$;

(ii) if $\lambda_{P T}>\lambda_{P T}^{*}\left(\lambda_{S}\right)$,

$$
\gamma\left(\lambda_{S}, \mathcal{A}_{P}\right)=\kappa \cdot\left(\frac{1-\beta_{0}^{\bar{\psi}}}{\beta_{0}^{\bar{\psi}}}+\tau\right)
$$


THEOREM 6. For a RCM Network $\mathcal{G}_{t}\left(\lambda_{S} ; f(r) ; \mathcal{A}_{P}\right)$ with $\lambda_{S}$ $>\lambda_{c}(f(r))$, the conclusion on $\gamma\left(\lambda_{S}, \mathcal{A}_{P}\right)$ in Theorem 5 still holds.

THEOREM 7. For a BM Network $\mathcal{G}_{t}\left(\lambda_{S} ; h(r) ; \mathcal{A}_{P}\right)$, with density $\frac{\lambda_{c}(h(r))}{\mathcal{R}^{2}}<\lambda_{S}<\lambda_{c}(h(r))$, we have:

$$
\gamma\left(\lambda_{S}, \mathcal{A}_{P}\right) \geq \frac{1}{\beta_{0}^{\eta} \cdot E\left[\min \left\{\mathcal{M}_{h(r)}\left(\lambda_{S}, \mathcal{A}_{P}\right), \frac{r_{s}}{\tau}\right\}+r_{s}\right]},
$$

where $\eta$ is a constant satisfying

$$
\left\lfloor\pi R_{p}^{2} \lambda_{P T}\right\rceil<\eta \leq\left\lfloor\pi\left(\frac{\min \left\{\mathcal{M}_{h(r)}\left(\lambda_{S}, \mathcal{A}_{P}\right), \frac{r_{s}}{\tau}\right\}}{2}+R_{p}\right)^{2} \lambda_{P T}\right\rceil .
$$

Proof. It is clear than $E\left[\mathcal{T}_{p}\left(l_{k}\right)\right]=\tau$, then item $(i)$ of Theorem 5 can be proved using Lemma 3. Item (ii) is also straightforward. We can obtain it by simply adding a constant $\tau$ in the results of Theorem 2.

The proof of Theorem 7 is similar to that of Theorem 4 . The only difference is the constraint on the physical size of $\mathcal{M}_{h(r)}\left(\lambda_{S}, \mathcal{A}_{P}\right)$. The largest range of one connected cluster is less than $\frac{r_{s}}{\tau}$ since the largest distance that the message can be forwarded is $\frac{r_{s}}{\tau}$ in one slot,. Therefore, the cluster size is $\min \left\{\mathcal{M}_{h(r)}\left(\lambda_{S}, \mathcal{A}_{P}\right), \frac{r_{s}}{\tau}\right\}$. Then we have:

$$
\begin{aligned}
\left\|u_{m+1}-u_{m}\right\| & \leq\left\|u_{m}-w_{m}\right\|+\left\|u_{m+1}-w_{m}\right\| \\
& \leq \min \left\{\mathcal{M}_{h(r)}\left(\lambda_{S}, \mathcal{A}_{P}\right), \frac{r_{s}}{\tau}\right\}+r_{s}
\end{aligned}
$$

By iteration of this inequalities, we obtain

$$
\begin{aligned}
d(u, v) & \leq \sum_{m=0}^{M-1}\left\|u_{m+1}-u_{m}\right\|+\left\|u_{M}-v\right\| \\
& \leq \sum_{m=0}^{M}\left(\min \left\{\mathcal{M}_{h(r)}\left(\lambda_{S}, \mathcal{A}_{P}\right), \frac{r_{s}}{\tau}\right\}+r_{s}\right) .
\end{aligned}
$$

Using the same method in previous section, we immediately get

$$
\begin{aligned}
\gamma\left(\lambda_{S}, \mathcal{A}_{P}\right) & =\frac{\mathcal{T}_{P}(\Upsilon)+\mathcal{T}_{S}(\Upsilon)}{d(u, v)} \\
& \geq \frac{1}{\beta_{0}^{\eta} \cdot E\left[\min \left\{\mathcal{M}_{h(r)}\left(\lambda_{S}, \mathcal{A}_{P}\right), \frac{r_{s}}{\tau}\right\}+r_{s}\right]}
\end{aligned}
$$

Then the proof of Theorem 7 is finished.

\section{SIMULATIONS}

In this section, we will present simulation results to verify our theoretical analysis of $\gamma\left(\lambda_{S}, \mathcal{A}_{P}\right)$. To begin with, we study the three critical densities for percolation, $\lambda_{c}(h(r)), \lambda_{c}(f(r))$, and $\lambda_{P T}^{*}\left(\lambda_{S}\right)$. Then we investigate the two constants $\bar{\psi}$ and $\kappa$, which have been defined in Equation (4) and Lemma 3, respectively. Finally, we present the simulation results of $\gamma\left(\lambda_{S}, \mathcal{A}_{P}\right)$ in Theorems 2 and 3 . However, due to the space limitation, we neglect the simulation for Theorem 4 and the impact of propagation delay.

According to continuum percolation theory, there exists a phase transition in their connectivity property: $\mathcal{C}_{\mathcal{O}}^{0}$ has infinite nodes when $\lambda_{S}>\lambda_{c}(h(r))$. Our simulation results verified the phase transition of secondary network in both BM and RCM model. In Figure 4 , we can conclude that $\lambda_{c}(h(r)) \approx 1.03$ and $\lambda_{c}(f(r)) \approx 1.63$. The presence of a tail in the result is due to the finiteness of simulation process. ${ }^{12}$ Lemma 1 is also verified by the simulation result.

\footnotetext{
${ }^{12}$ Our simulation parameters are $r_{s}=1.40, R_{p}=2.87$. Since primary users tend to use continuous slots to finish transmission, we set $p_{11}=0.75$ and $p_{00}=0.9 . \mathbb{P}_{P T}(t), \mathbb{P}_{P R}(t)$ and $\mathbb{P}_{S}(t)$ are distributed in a square $[-20,20] \times[-20,20]$ according to homogeneous Poisson Point Process.
}

When $\lambda_{P T}<\lambda_{P T}^{*}\left(\lambda_{S}\right)$, the secondary network will be comsupercritical, which implies that $\mathcal{C}_{\mathcal{O}}^{0}$ still contains infinite secondary nodes, i.e. the unique infinite connected component $\mathcal{C}_{\mathcal{O}}$ still exists, in spite of the activities of primary network. However, $\mathcal{C}_{\mathcal{O}}$ is broken into mutually disconnected finite clusters when $\lambda_{P T}>\lambda_{P T}^{*}\left(\lambda_{S}\right)$. In Figure 5, simulation result shows that the critical primary density $\lambda_{P T}^{*}(2.4) \approx 0.03$.
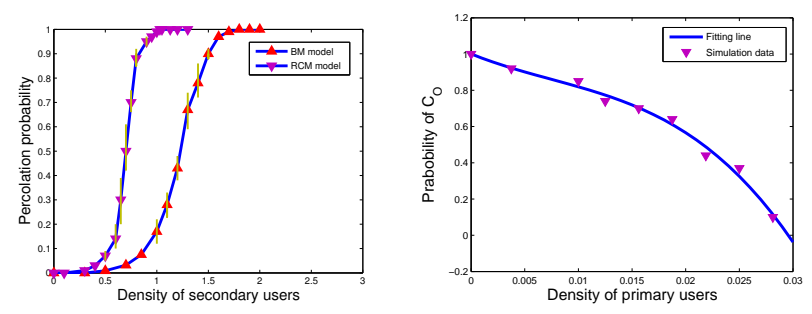

Figure 4: Critical density of Figure 5: Critical density of secondary user for percola- primary user for percolation: tion in BM and RCM model: $\lambda_{P T}^{*}\left(\lambda_{S}\right)$. It is clear that when $\lambda_{c}(h(r)), \lambda_{c}(f(r))$. We can $\lambda_{P T}>0.03$, the infinite consee that $\lambda_{c}(h(r)) \approx 1.03$ and nected component $\mathcal{C}_{\mathcal{O}}$ is broken $\lambda_{c}(f(r)) \approx 1.63$. The tail in into mutually disconnected fithe figure is due to the finite- nite clusters almost surly. ness of simulation.

According to Lemma 2, $\bar{\psi}$ is a constant that features the number of primary users within the interfered region $\mathcal{I}\left(l_{k}\right)$. In Figure 6, we study $\bar{\psi}$ of two hundred links in one path and the simulation result shows that $\bar{\psi} \approx 3$, well between the lower and upper bound given in Equation (4).

In Lemma 3, we show that $\kappa$, describing the scaling behavior of hops number in one path, is a constant independent on $\lambda_{S}$. From Figure 7 , we can see $\kappa \approx 0.79$ for both BM and RCM model, which also verifies our Lemma 5 . Note that when $\lambda_{S}$ is small, $\kappa$ is relatively large. This is due to that Lemma 3 holds only when the network is supercritical. For the subcritical network ( $\lambda_{S}$ is less than critical density), $\kappa$ may not be a constant.
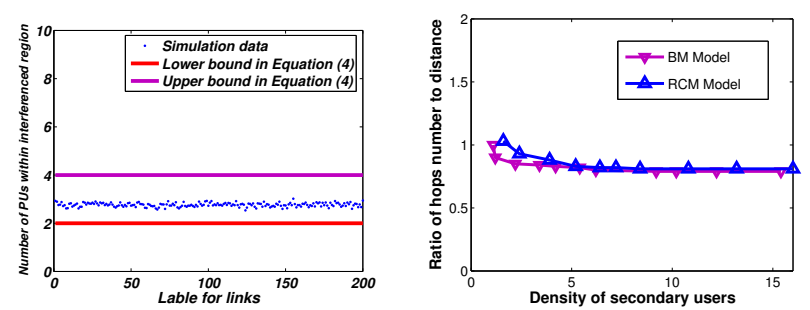

Figure 6: Average number of Figure 7: Ratio of hops number primary transmitters in inter- to distance in one path: $\kappa$. It is fered region $\mathcal{I}\left(l_{k}\right): \bar{\psi}$. Given clear that $\kappa$ is a constant inde$\lambda_{P T}=0.09375$, it is clear that pendent on $\lambda_{S}$ in supercritical $\bar{\psi}$ is a constant and $\bar{\psi} \approx 3$. network.

Now we are ready to present the simulation results for $\gamma\left(\lambda_{S}, \mathcal{A}_{P}\right)$. In Figure 8 , it is clear that $\gamma\left(\lambda_{S}, \mathcal{A}_{P}\right)$ converges to a constan$\mathrm{t}$ when distance is sufficiently large. The simulation parameters are $\lambda_{S}=2.4, \lambda_{P T}=0.035$ and the corresponding $\bar{\psi} \approx 1$. According to Theorem 2 , we have $\gamma\left(\lambda_{S}, \mathcal{A}_{P}\right) \approx 0.316$, which is roughly the same as our simulation result. Figure 9 illustrates the $\gamma\left(\lambda_{S}, \mathcal{A}_{P}\right)$ for different $\lambda_{P T}$. Simulation results also fit our analysis well. 


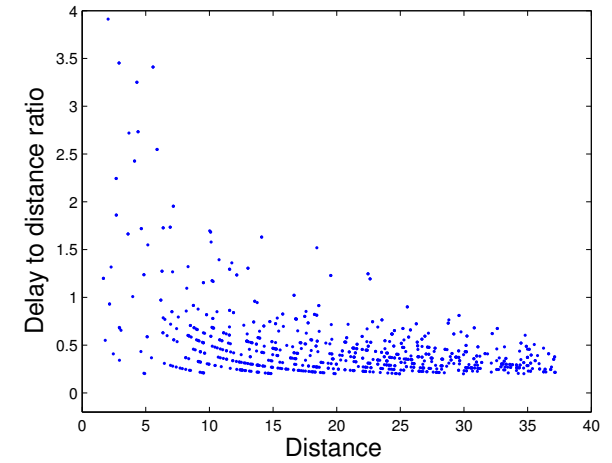

Figure 8: Illustration for Theorem 2. It is true that $\gamma\left(\lambda_{S}, \mathcal{A}_{P}\right)$ converges to a constant as the distance increases. Given $\lambda_{P T}=$ $0.035, \gamma\left(\lambda_{S}, \mathcal{A}_{P}\right) \approx 0.3$.

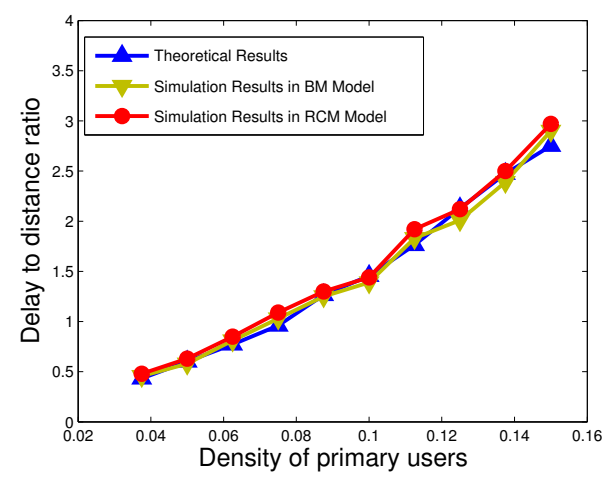

Figure 9: Illustration of $\gamma\left(\lambda_{S}, \mathcal{A}_{P}\right)$ for different $\lambda_{P T}$. It is clear that $\gamma\left(\lambda_{S}, \mathcal{A}_{P}\right)$ increases with respect to $\lambda_{P T}$.

\section{CONCLUSION AND DISCUSSION}

In this paper, we have studied the transmission delay in large scale ad hoc CR networks by analyzing the ratio of delay to distance $\gamma\left(\lambda_{S}, \mathcal{A}_{P}\right)$ as the distance goes to infinite. $\gamma\left(\lambda_{S}, \mathcal{A}_{P}\right)$ in three criteria of CR networks have been investigated, among which we present exact value of $\gamma\left(\lambda_{S}, \mathcal{A}_{P}\right)$ in both supercritical BM and RCM networks and a lower bound of $\gamma\left(\lambda_{S}, \mathcal{A}_{P}\right)$ in subcritical B$\mathrm{M}$ network. We proved that for com-supercritical CR networks, $\gamma\left(\lambda_{S}, \mathcal{A}_{P}\right)=0$, and $\gamma\left(\lambda_{S}, \mathcal{A}_{P}\right)=\kappa \cdot \frac{1-\beta_{0}^{\bar{\psi}}}{\beta_{0}^{\bar{\psi}}}$ for both the topsupercritical $\mathcal{G}_{t}\left(\lambda_{S} ; h(r) ; \mathcal{A}_{P}\right)$ and $\mathcal{G}_{t}\left(\lambda_{S} ; f(r) ; \mathcal{A}_{P}\right)$. Moreover, we showed $\gamma\left(\lambda_{S}, \mathcal{A}_{P}\right)>\frac{1}{\beta_{0}^{\eta} \cdot E\left[\mathcal{M}_{h(r)}\left(\lambda_{S}, \mathcal{A}_{P}\right)+r_{s}\right]}$ in the subcritical network $\mathcal{G}_{t}\left(\lambda_{S} ; h(r) ; \mathcal{A}_{P}\right)$. Finally, we use simulation results to verify our theoretical analysis.

As to the future work, one of the main challenges is analyzing $\gamma\left(\lambda_{S}, \mathcal{A}_{P}\right)$ in subcritical RCM network. Because we have to prove that the message can also have a probability to be forwarded in a subcritical RCM network under mobility models, which needs more intensive and in-depth study of continuum percolation theory. Furthermore, we hope to find a more precise description of $\gamma\left(\lambda_{S}, \mathcal{A}_{P}\right)$ in subcritical BM network in our future works. Finally, network capacity or throughput has not been discussed in this pa- per. Our future works hope to find the capacity and delay tradeoff in CR networks.

\section{Acknowledgment}

This paper is supported by National Fundamental Research Grant (No. 2011CB302701); NSF China (No. 60832005); China Ministry of Education New Century Excellent Talent (No. NCET-100580); China Ministry of Education Fok Ying Tung Fund (No. 122002); Qualcomm Research Grant; Shanghai Basic Research Key Project (No. 11JC1405100).

\section{REFERENCES}

[1] R. Meester and R. Roy, "Continuum Percolation," NewYork: Cambridge University Press, 1996.

[2] T. Liggett, "An Improved Subadditive Ergodic Theorem," Annals of Prob., vol. 13, pp. 1279-1285, 1985.

[3] J. F. C. Kingman, "Poisson Processes," Clarendon Press, Oxford, 1993.

[4] Federal Communications Commission Spectrum Policy Task Force, "Report of the spectrum efficiency working group," Nov. 2002.

[5] P. Gupta and P. R. Kumar, "The capacity of wireless networks," IEEE Trans. on Information Theory, vol. 46, no. 2, pp. 388-404, 2000.

[6] P. Gupta and P. R. Kumar, "Critical Power for Asymptotic Connectivity in Wireless Networks," in Stochastic Analysis, Control, Optimization and Applications: A Volume in Honor of W.H. Fleming, W. M. McEneany, G. Yin, Q. Zhang, Eds. Boston, MA: Birkhauser, pp. 547-566, 1998.

[7] C. F. Huang, and Y. C. Tseng, "The coverage problem in a wireless sensor network," in Mobile Networks and Applications, vol. 10, no. 4, pp. 519-528, 2005.

[8] C. Bettstetter, "On the minimum node degree and connectivity of a wireless multihop network," in Proceedings of the $3 \mathrm{rd}$ ACM international symposium on Mobile ad hoc networking and computing, pp. 80-91, 2002.

[9] L. Ding, T. Melodia, S. Batalama, J. Matyjas, M. Medley, "Cross-layer Routing and Dynamic Spectrum Allocation in Cognitive Radio Ad Hoc Networks, " in IEEE Transactions on Vehicular Technology, vol. 59, no. 4, pp. 1969-1979, May 2010

[10] P. Wang, and I. F. Akyildiz, A. M. and Al-Dhelaan, "Dynamic connectivity of cognitive radio ad-hoc networks with time-varying spectral activity," in Proceedings of Globecom, 2010

[11] W. Ren, Q. Zhao, and A. Swami, "Connectivity of heterogeneous wireless networks," in Information Theory, IEEE Transactions on, vol. 57, no. 7, pp. 4315-4332, 2011.

[12] S. Bodas, S. Shakkottai, L. Ying and R. Srikant, "Scheduling for Small Delay in Multi-rate Multi-channel Wireless Networks," in Proceedings of IEEE Infocom, Shanghai, China, 2011

[13] Z. Kong and E. M. Yeh, "On the Latency for Information Dissemination in Mobile Wireless Networks," in Proc. ACM MobiHoc'08, Hong Kong SAR, China, May. 2008.

[14] S. Zhao, L. Fu, X. Wang, Q. Zhang, "Fundamental Relationship between Node Density and Delay in Wireless Ad Hoc Networks with Unreliable Links," in ACM MobiCom 2011, Sept. 2011. 